\title{
Pattern Formation in Catalyzed Surface Reactions Studied by In Situ SEM
}

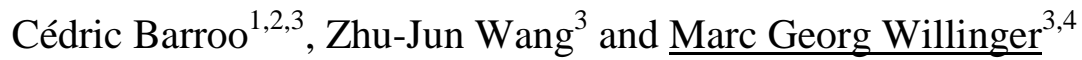

${ }^{1 .}$ Chemical Physics of Materials and Catalysis, Université libre de Bruxelles, Belgium.

2. Interdisciplinary Center for Nonlinear Phenomena and Complex Systems (CENOLI), Université libre de Bruxelles, Belgium.

3. Department of Inorganic Chemistry, Fritz Haber Institute of the Max Planck Society, Germany.

${ }^{4 .}$ Scientific Center for Optical and Electron Microscopy (ScopeM), ETH Zurich, Switzerland.

Working catalysts correspond to systems that are operated far from thermodynamic equilibrium. The state of the active catalyst therefore differs from what is observed ex situ. Structure-function relationships should thus be studied under relevant reaction conditions. While in situ TEM enables observation of atomistic details under conditions of slow kinetics, in situ SEM delivers complementary information about catalyst dynamics at reduced lateral resolution. It provides insight about collective phenomena that are controlled by heat and mass transport and enables a multi-scale approach for the study of complex systems under gas pressures ranging from $10^{-4}$ to $10^{3} \mathrm{~Pa}$ and temperatures up to $1500^{\circ} \mathrm{C}$.

Recently, direct observation of graphene growth by chemical vapor deposition inside the chamber of an environmental SEM (ESEM) demonstrated the high sensitivity of the secondary electron signal to changes in the surface coverage and work function of metal catalysts. [1,2] The ability to directly visualize the formation of monolayers of carbon on a $1000^{\circ} \mathrm{C}$ hot surface suggested that the system might be sensitive enough to differentiate work function changes due to gas adsorption. In order to test this, we imaged Pt surfaces during the reaction of $\mathrm{NO}_{2}$ with $\mathrm{H}_{2}$ by in situ SEM.

Nitrogen oxides $\left(\mathrm{NO}_{\mathrm{x}}\right)$ are produced during the combustion of fuels in lean-burn vehicles. Selective Catalytic Reduction (SCR) of $\mathrm{NO}_{\mathrm{x}}$ is one of the options to reduce $\mathrm{NO}_{\mathrm{x}}$ emissions. Currently, ammonia injection is considered the method of choice to meet legislative target levels. An alternative would be to use hydrogen, which could be produced through on-board reforming. One of the advantages of $\mathrm{H}_{2}$-SCR would be the relatively low operating temperatures so as to improve the vehicle cold start behavior. Platinum-Group-Metals (PGM's) may be anticipated to act as suitable catalysts for high $\mathrm{NO}_{\mathrm{x}}$ conversion in $\mathrm{H}_{2}$-SCR. However, little is known about the elemental mechanisms and microkinetics of this reaction. Our research aims at filling this gap in knowledge and to establish reliable structure-function relationships.

In situ experiments were performed in a modified commercial ESEM (FEI Quanta 200) equipped with a heating stage, gas feeding system and mass spectrometry for analysis of the gas composition and detection of reaction products. Pt foils were cleaned ex situ, and then annealed under hydrogen prior to exposure to reactive mixtures of $\mathrm{NO}_{2}$ and $\mathrm{H}_{2}$ at pressures ranging from $10^{-3}$ to $20 \mathrm{~Pa}$. Previously performed experiments on a single nanoparticle of Pt by field emission techniques served as a guideline for the interpretation of work function changes during the reaction. [3,4]

The introduction of pure $\mathrm{NO}_{2}$ in the ESEM chamber reduces the brightness due to the increasing work function of $\mathrm{Pt}$ in presence of $\mathrm{NO}_{2}$ (Figure 1). After addition of $\mathrm{H}_{2}$, nonlinear behaviors in the form of dynamic reaction diffusion processes and spiral patterns can be observed (Figure 2) for specific reaction conditions. A brightness analysis of the reaction pattern allows to distinguish 3 different brightness levels on a single propagating wave. This is not only the proof that ESEM is indeed a surface-sensitive technique that can be used to probe gas-surface interactions, but also that ESEM can differentiate different adsorbates species as a function of their effect on the work function. Furthermore, it is possible to directly compare the reactivity of differently oriented Pt grains and the structure sensitivity of the reaction, the spillover between different facets, and how the presence of 
boundaries and defects affect the reactivity. In situ SEM can thus be considered as surface science method with surface sensitivities comparable to photoemission electron microscopy (PEEM), but with less restrictions regarding the structure of the sample and the advantage of a higher lateral resolution and the ability to observe surface dynamics over a large pressure range. [5]

\section{References:}

[1] Z-J Wang et al, Nat Comm 7 (2016), 13256.

[2] MG Willinger et al, Microsc Microanal 22 (Suppl 3) (2016), 784.

[3] C Barroo et al, Appl Surf Sci 304 (2014), 2.

[4] C Barroo et al, Phys Rev Lett 117 (2016), 144501.

[5] CB thanks the Fonds de la Recherche Scientifique (F.R.S.-FNRS) and the Wallonie-Bruxelles International (Excellence grant WBI.WORLD) for financial support.
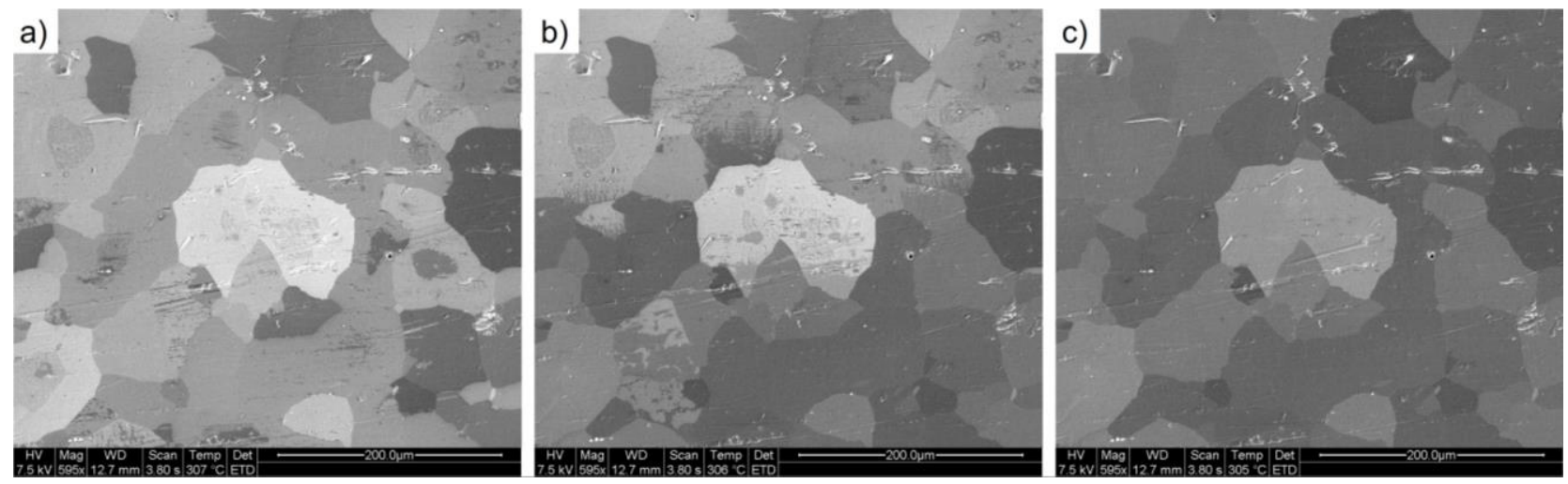

Figure 1. Changes in brightness during the adsorption of $\mathrm{NO}_{2}$ on $\mathrm{Pt}$ foil at $\approx 300^{\circ} \mathrm{C}$. Scale bar: $200 \mu \mathrm{m}$.
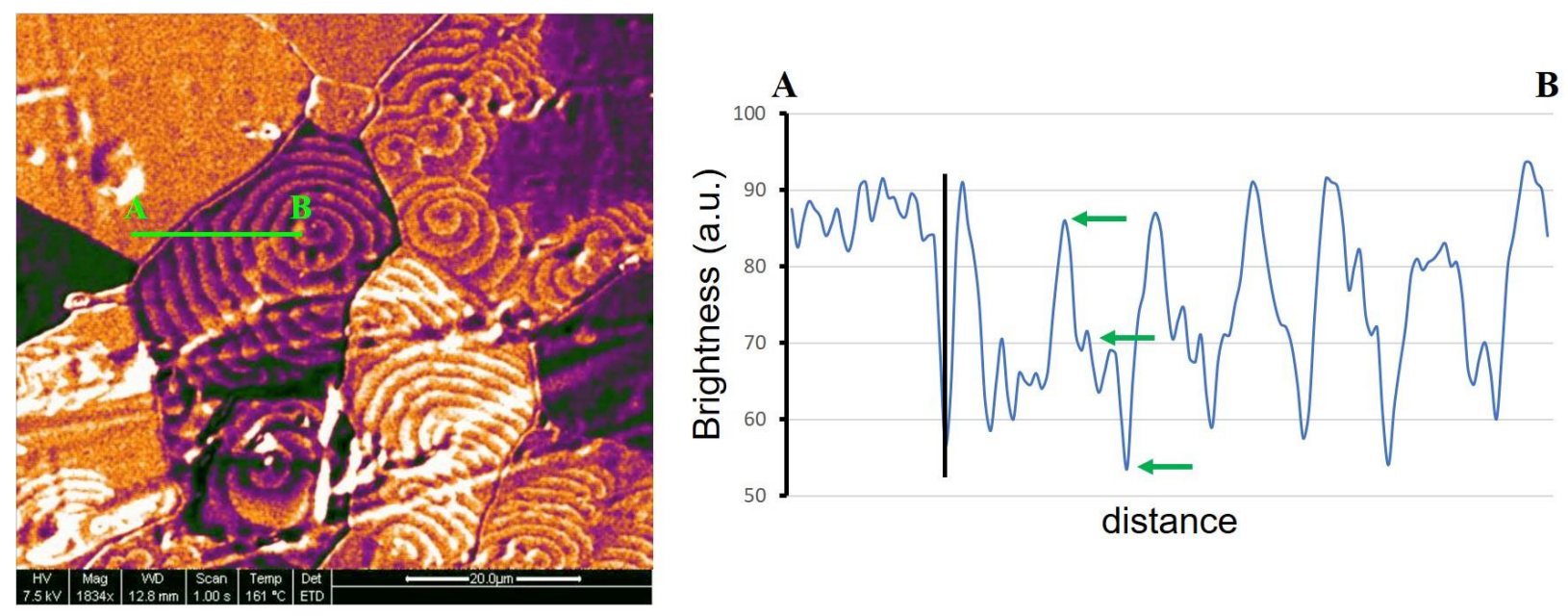

Figure 2. (left) ESEM snapshot during spiral pattern formation in the $\mathrm{NO}_{2}+\mathrm{H}_{2}$ reaction on Pt foil. (right) Brightness analysis showing three distinct brightness levels on a single propagating wave (marked as three arrows) and proving the sensitivity of the ESEM to different adsorbate-species. Scale bar: $20 \mu \mathrm{m}$. 